\title{
Factors Affecting Brain Metabolism Measured with ${ }^{18}$ FDG
}

\author{
Hongyun Zhu' ${ }^{1}$, Michael L. Goris ${ }^{2}$ \\ ${ }^{1}$ Department of Radiology, University of Arkansas for Medical Sciences (UAMS), Little Rock, USA \\ ${ }^{2}$ Division of Nuclear Medicine, School of Medicine, Stanford University, Stanford, USA \\ Email: mlgoris@stanford.edu
}

Received December 2, 2011; revised January 26, 2012; accepted February 8, 2012

\begin{abstract}
An observational finding found a large variation in the brain SUV in patients with multiple myeloma undergoing $\mathrm{PET} / \mathrm{CT}$. The first hypothesis considered a toxic effect of chemotherapeutic agents, but no correlation was found with hematological signs of toxicity. Low brain FDG uptake has been described with anesthesia, but this was not relevant in this case. An alternative is the presence of a large FDG avid mass, but that was excluded. Since there was a question of chemotherapy toxicity, the metrics used for comparison were Hemoglobin levels (Hgb, g/dl), Erythrocyte count (RBC, $\mathrm{M} / \mu \mathrm{L}$ ), Lymphocytes absolute counts (Lymph\#, K/ $\mu \mathrm{L}$ ) and \% (lymph, \%), Granulocytes Neutrophils, K/ $\mu \mathrm{L}$ ), age and C-reactive protein levels (CRP, g/L). The liver SUV (standardized uptake value) was included to eliminate unexpected global effects on the SUV values, since FDG uptake is a competitive system with a single source (plasma FDG levels). There was in fact no correlation between brain SUV and hepatic SUV, eliminating the so-called super scan effect. Further analysis, however, revealed a strong positive correlation with hemoglobin or RBC levels, but an inverse effect with Neutrophils, C-reactive proteins and age (in years). The results suggest that brain metabolism strongly depends on oxygen supply and may be depressed by general inflammatory diseases and independently with age. If the variation of glucose metabolism correlates with cognitive deficits (CD), considering general measures of good health may be a first step for relief of age related $\mathrm{CD}$.
\end{abstract}

Keywords: Brain 18-FDG Uptake; Anemia; Inflammation

\section{Introduction}

An observational finding found a large variation in the brain SUV (standardized uptake value) in patients with multiple myeloma undergoing PET/CT. The first hypothesis considered a toxic effect of chemotherapeutic agents, but no correlation was found with hematological signs of toxicity. Low brain FDG uptake has been described with anesthesia, but this was not relevant in this case. An alternative is the presence of a large FDG avid mass, but that was excluded.

Since there was a question of chemotherapy toxicity, the metrics used for comparison were Hemoglobin levels

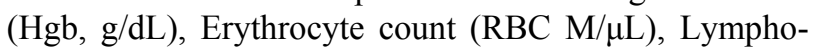
cytes absolute counts (Lymph\#, K/ $/ \mathrm{L}$ ) and \% (lymph, \%), Granulocytes (Neutrophils K/ $\mu \mathrm{L}$ ), age (in years) and Creactive protein levels $(\mathrm{CRP}, \mathrm{g} / \mathrm{L})$. The liver $\mathrm{SUV}$ was included to eliminate unexpected global effects on the SUV values, since FDG uptake is a competitive system with a single source (plasma FDG levels).

\section{Materials and Methods}

There were 40 patients, ( $70 \%$ male), average age 60.7 (range 44-83), with 173 FDG PET/CT scans. The reason for the study was evaluation of Multiple Myeloma ther- apy. Patients were routinely followed with the metrics cited above. Brain SUV was measured in the central brain cortex, but the value was not site sensitive, since the correlation between central and visual was 0.95 and between central and nuclei was 0.96 . In addition, patients with abnormal cerebral regional FDG distributions (e.g. as in Alzheimer's, Fronto-parietal dementia, multi-stroke dementia) were not included. The central brain activity in SUV units was on average $4.96 \pm 1.84$ (range 1.5 - 9.7). The average glucose level was $134 \pm 27 \mathrm{mg} / \mathrm{dl}$ (range 104198).

After the FDG injection, the patients were kept in a quiet, dimly lighted room, for $1 \mathrm{hr}$, before imaging began.

The analysis consisted first in an evaluation of the intercorrelation of the metric listed above prior to using multiple regressions. Multiple regressions were performed first with all metrics and later only with those not positively inter-correlated or with a significant single regression with brain SUV.

\section{Results}

Central brain SUV correlated significantly negative with age $(\mathrm{p}<0.001)$, CRP $(\mathrm{p}<0.05)$ and Neutrophils $(\mathrm{p}<$ 
$0.01)$ and significantly positive with RBC (p $<0.001)$, $\operatorname{Hgb}(\mathrm{p}<0.001), \operatorname{lymph} \%(\mathrm{p}<0.005)$ and Lymph\# $(\mathrm{p}<$ $0.05)$.

There was significant negative correlation between WBC and liver SUV $(\mathrm{p}<0.05)$ and between WBC and lymph\% $\%(p<0.001)$. However, the linear regression between WBC and brain SUV was not significant $(\mathrm{p}>$ 0.05). Lymph\% correlated positively with RBC $(\mathrm{p}<$ $0.001)$ and $\mathrm{Hgb}(\mathrm{p}<0.001)$.

The results was that multiple regressions were performed only for HGB (not RBC), Age, Neutrophils, lymph\# and CRP. The liver SUV was included to test for a super-scan effect.

The results of single regression between the various metric and brain SUV with their $\mathrm{p}$ values are shown in Table 1.

Table 2 shows serial multiple regressions in which the significant factors from the previous multiple regressions are withdrawn.

\section{Discussion}

Recent and remote chemotherapy could influence brain uptake. However, the fact that a potential effect of chemotherapy would vary in the subject is not relevant in this study. First, it is unlikely to be correlated with age. Second, the effect on bone marrow, if there was one, would be a decrease in both red and white blood elements. What we observe is an opposite effect on brain uptake of RBC and Neutrophils. Age by itself did not correlate with the next 2 important factors, the level of hemoglobin or red cell counts, or the level of neutrophils.

Diffuse low glucose metabolism measured with FDG has been described in Halothane anesthesia [1], in low states of consciousness [2], and paradoxically with low blood glucose [3]. In addition it has been observed in diffuse brain injury [4] without large focal abnormalities. An alternative explanation is due to a sink effect, if other structures or pathologies are very FDG avid. They would then act as a FDG sink, since all structures in the body accumulate FDG from the same plasma pool. This phenomenon has been described as a metabolic super scan [5].

None of those factors was present in this population. The lack of correlation between brain and liver SUV excludes the metabolic super scan. Glucose levels in the plasma were within limit (above $6 \mathrm{mM}$ ). There was no (generalized) history of trauma or low consciousness.

\section{Conclusion}

Non-neurological factors (anemia, age, inflammation) seem to adversely affect FDG uptake in the brain. Those effects are independent of age, and, if shown to be causative, would suggest methods of therapeutic interventions.
Table 1. Negative effects are age, CRP, Neutrophils and WBC (not significant). Except for age they are all associated with inflammation. Positive effects are $\mathrm{Hgb}$ and RBC, lymph\#, and lymph\%. There is no significant correlation between brain and liver SUV.

\begin{tabular}{ccc}
\hline & Slope & $\mathrm{p}<$ \\
\hline Age & -0.048 & 0.001 \\
HgB & 0.376 & 0.001 \\
Lymph\# & 0.368 & 0.001 \\
CRP & -0.011 & 0.033 \\
Neutro & -0.077 & 0.018 \\
Liver & 0.244 & 0.600 \\
WBC & -0.061 & 0.059 \\
RBC & 1.093 & 0.001 \\
Lymph\% & 0.026 & 0.007 \\
\hline
\end{tabular}

Table 2. With the uncorrelated metrics if all are used in multiple regressions, Age and $\mathrm{Hgb}$ are significant. If those are eliminated, Lymph\# and Neutrophils are significant. The next significant factor is the CRP. The liver does not reach significance. The $F$-ratio is the ratio of the variance in brain SUV explained by the regression over the residual variance in brain SUV.

\begin{tabular}{cccc}
\hline Metric & \multicolumn{3}{c}{$\mathrm{p}$ value } \\
\hline Hemoglobin & $1.1 \mathrm{E}-05$ & & \\
Age & 0.0001 & & \\
Neutro & 0.0760 & 0.0295 & \\
Lymph & 0.3103 & 0.0362 & 0.0346 \\
CRP & 0.950 & 0.0560 & 0.5388 \\
Liver & 0.8122 & 0.7997 & 0.089 \\
F-Ratio & $1.72 \mathrm{E}-08$ & 0.007189 & \\
\hline
\end{tabular}

If the low FDG uptake is associated with cognitive deficit, it may be that improving those general dysfunctions (except for age), may be the first step and easiest therapeutic approach.

\section{REFERENCES}

[1] M. T. Alkire, C. J. Pomfrett, R. J. Haier, M. V. Gianzero, C. M. Chan, B. P. Jacobsen and J. H. Fallon "Functional Brain Imaging during Anesthesia in Humans: Effects of Halothane on Global and Regional Cerebral Glucose Metabolism," Anesthesiology, Vol. 90, No. 3, 1999, pp. 701709. doi:10.1097/00000542-199903000-00011

[2] M. Boly, C. Phillips, L. Tshibanda, A. Vanhaudenhuyse, 
M. Schabus, T. T. Dang-Vu, G. Moonen, R. Hustinx, P. Maquet and S. Laureys, "Intrinsic Brain Activity in Altered States of Consciousness," Annals of the New York Academy of Sciences, Vol. 1129, 2008, pp. 119-129. doi:10.1196/annals.1417.015

[3] R. Meierhans, M. Béchir, S. Ludwig, J Sommerfeld, G. Brandi, C. Haberthür, R. Stocker and J. F. Stover, "Brain Metabolism Is Significantly Impaired at Blood Glucose below $6 \mathrm{mM}$ and Brain Glucose below $1 \mathrm{mM}$ in Patients with Severe Traumatic Brain Injury," Critical Care, Vol. 14, No. 1, 2010, p. R13. doi:10.1186/cc8869

[4] N. Nakayama, A. Okumura, J. Shinoda, T. Nakashima and T. Iwama, "Relationship between Regional Cerebral Metabolism and Consciousness Disturbance in Traumatic Diffuse Brain Injury without Large Focal Lesions: An FDG-PET Study with Statistical Parametric Mapping Analysis," Journal of Neurology, Neurosurgery \& Psychiatry, Vol. 77, No. 7, 2006, pp. 856-862. doi:10.1136/jnnp.2005.080523

[5] D.-W. Kim, C. G. Kim, S.-A. Park, S.-A. Jung and S-H. Yang, "Metabolic Super Scan in 18F-FDG PET/CT Imaging," Journal of Korean Medical Science, Vol. 25, No. 8, 2010, pp. 1256-1257. doi:10.3346/jkms.2010.25.8.1256

\section{Supporting Data}

Table S1. Inter-correlation between metrics and brain SUV.

\begin{tabular}{|c|c|c|c|c|c|c|c|c|c|c|}
\hline & Brain frontal & Age & Liver & WBC & RBC & Hemoglobin & Lymph\% & Lymph\# & CRP & Neutro \\
\hline Brain frontal & 1 & & & & & & & & & \\
\hline Age & -0.2747 & 1 & & & & & & & & \\
\hline Liver & 0.047734 & 0.021864 & 1 & & & & & & & \\
\hline WBC & -0.14381 & 0.132306 & -0.1327 & 1 & & & & & & \\
\hline $\mathrm{RBC}$ & 0.33053 & 0.03899 & -0.00581 & 0.052584 & 1 & & & & & \\
\hline Hemoglobin & 0.37794 & 0.010832 & 0.05235 & 0.014243 & 0.91785 & 1 & & & & \\
\hline Lymph\% & 0.205287 & 0.079511 & 0.119979 & -0.29681 & 0.121782 & 0.198546 & 1 & & & \\
\hline Lymph\# & 0.167963 & 0.068117 & 0.029356 & 0.173632 & 0.304025 & 0.320882 & 0.662159 & 1 & & \\
\hline CRP & -0.16137 & 0.006303 & -0.00727 & 0.050013 & -0.23132 & -0.26407 & -0.08514 & -0.05294 & 1 & \\
\hline Neutro & -0.17894 & 0.120962 & -0.14047 & 0.981173 & -0.00624 & -0.04847 & -0.43118 & -0.01984 & 0.061156 & 1 \\
\hline
\end{tabular}

Inter-correlation of brain SUV and the metrics used in this paper. The yellow background highlights the negative correlations.

Table S2. The significance of the correlation shown in Table S1 is not shown if $p>0.05$.

\begin{tabular}{|c|c|c|c|c|c|c|c|c|c|c|}
\hline & Brain frontal & Age & liver & WBC & RBC & Hemoglobin & Lymph\% & Lymph\# & CRP & Neutro \\
\hline \multicolumn{11}{|l|}{ Brain frontal } \\
\hline Age & 0.001 & & & & & & & & & \\
\hline \multicolumn{11}{|l|}{ liver } \\
\hline WBC & 0.05 & 0.05 & 0.05 & & & & & & & \\
\hline $\mathrm{RBC}$ & 0.001 & & & & & & & & & \\
\hline Hemoglobin & 0.001 & & & & 0.001 & & & & & \\
\hline Lymph\% & 0.005 & & & 0.001 & & 0.005 & & & & \\
\hline Lymph\# & 0.05 & & & 0.05 & 0.001 & 0.001 & 0.001 & & & \\
\hline CRP & 0.05 & & & & 0.005 & 0.001 & & & & \\
\hline Neutro & 0.01 & & 0.05 & 0.001 & & & 0.001 & & & \\
\hline
\end{tabular}

Brain central activity correlated negatively with Age $(\mathrm{p}<0.001)$, WBC $(\mathrm{p}<0.05)$, CRP $(\mathrm{p}<0.05)$, and Neutrophils (Neutro) $(\mathrm{p}<0.01)$, but positively with RBC $(\mathrm{p}<0.01)$, Hemoglobin $(\mathrm{Hgb})(\mathrm{P}, 0.01)$, lymphocytes \% $($ lymph\%) $(\mathrm{p}<0.005)$ and lymphocytes absolute numbers $($ Lymph\#) $(\mathrm{p}<0.05)$. 
Table S3. Multple regressions using all metrics: significance.

\begin{tabular}{|c|c|c|c|c|c|c|c|c|c|}
\hline \multirow[t]{2}{*}{ Metric } & \multicolumn{9}{|c|}{ p-values } \\
\hline & 0.0002 & & & & & & & & \\
\hline Hemoglobin & 0.0735 & 0.0418 & & & & & & & \\
\hline $\mathrm{RBC}$ & 0.6784 & 0.9175 & 0.0000 & & & & & & \\
\hline Lymph \# & 0.2992 & 0.1389 & 0.0996 & 0.0234 & & & & & \\
\hline Lymph \% & 0.1201 & 0.4007 & 0.2476 & 0.4841 & 0.0251 & & & & \\
\hline Neutro & 0.1611 & 0.1000 & 0.0599 & 0.0503 & 0.9176 & 0.0312 & & & \\
\hline CRP & 0.6016 & 0.5178 & 0.3677 & 0.0831 & 0.2170 & 0.3262 & 0.0415 & & \\
\hline WBC & 0.1678 & 0.0931 & 0.0570 & 0.0492 & 0.8632 & 0.0317 & 0.0834 & 0.0834 & 0.0693 \\
\hline liver & 0.3891 & 0.7606 & 0.5700 & 0.1949 & 0.1277 & 0.0420 & 0.7020 & 0.7020 & 0.7037 \\
\hline F-ratio & 3.1E-09 & 5.1E-07 & $1.2 \mathrm{E}-06$ & 0.00226 & 0.009 & 0.036 & 0.048 & 0.048 & 0.15 \\
\hline
\end{tabular}

With all the metric included, the significant factor is age. All other metrics do not significantly contribute. If age is eliminated, the significant contribution is from hemoglobin. Next come RBC, then lymph\#, then lymph\% etc. Some factors contribute negatively (gray background). WBC and liver never contribute significantly. The F-ratio is the ratio of the contribution of the regression to the variance of the data over the residual variance. When only liver and WBC remain the F-ratio is not significant.

Table S4. Range of significant metrics.

\begin{tabular}{lcc}
\hline Metric & Means & Range \\
\hline Liver SUV & $2.19 \pm 0.36$ & $1.5-3.6$ \\
Hgb & $10.62 \pm 1.86$ & $6.8-17.2$ \\
Lymph\# & $1.00 \pm 0.84$ & $0.0024-5.44$ \\
CRP & $19.05 \pm 25.51$ & $0-148$ \\
Neutrophils & $4.11 \pm 4.30$ & $0.04-42.46$ \\
\hline
\end{tabular}

The table includes the metrics selected for multiple regressions.

Table S5. Individual correlations were: Table S5.

\begin{tabular}{lcc}
\hline & Slope & $\mathbf{p}<$ \\
\hline Age & -0.048 & 0.001 \\
HgB & 0.376 & 0.001 \\
Lymph\# & 0.368 & 0.001 \\
CRP & -0.011 & 0.033 \\
Neutro & -0.077 & 0.018 \\
Liver & 0.244 & 0.600 \\
WBC & -0.061 & 0.059 \\
RBC & 1.093 & 0.001 \\
Lymph\% & 0.026 & 0.007 \\
\hline
\end{tabular}

Table S6. Multiple regressions between all metrics and brain SUV.

\begin{tabular}{lccc}
\hline Metric & Slopes & Standard Error & p-value \\
\hline Hgb & 0.3332 & 0.0735 & 0.00001 \\
Age & -0.0472 & 0.0120 & 0.00012 \\
Intercept & 4.2009 & 1.2801 & 0.00126 \\
Neutro & -0.0528 & 0.0296 & 0.07603 \\
Lymph\# & 0.1594 & 0.1566 & 0.31030 \\
CRP & -0.0042 & 0.0049 & 0.39498 \\
liver & 0.0832 & 0.3496 & 0.81221 \\
\hline
\end{tabular}

When those (Table S6) metrics are used with their slope and the intercept, the predicted value $\mathrm{B}_{\mathrm{eSUV}}=4.2009-0.0472 \times$ age $-0.0528 \times$ Neutrophiles $+0.1594 \times$ lymphocytes $-0.0042 \times \mathrm{CRP}+0.0832 \times$ Liver $_{\text {SUV }}$ the prediction (expected) is highly significant and not significantly different from unity (Table S7)

Table S7. Prediction of brain SUV using all significant not intercorrelated metrics.

\begin{tabular}{lccc}
\hline & Coefficients & $\begin{array}{c}\text { Standard } \\
\text { Error }\end{array}$ & p-value \\
\hline Intercept & 0.0000 & 0.6781 & 1 \\
Expected & 1.0000 & 0.1343 & $4.4754 \mathrm{E}-12$ \\
F-ratio & & & $4.4754 \mathrm{E}-12$ \\
\hline
\end{tabular}

